\title{
Some Hematological Parameters In Malaria Parasitaemia
}

\author{
Okoroiwu Ijeoma Leticia ${ }^{1}$, Obeagu Emmanuel Ifeanyi ${ }^{2}$ and \\ Elemchukwu Queen ${ }^{3}$, Ochei Kingsley Chinedum ${ }^{4}$ \\ 1. Lecturer (P.hD), Department of Medical Laboratory Science,Faculty of Health Science,Imo State \\ University, Owerri,Nigeria. \\ 2. Diagnostic Laboratory,University Health services Department,Michael okpara University of \\ Agriculture, Umudike, Nigeria, \\ 3 Rivers State College of Health Science and Technology, Port Harcourt \\ 4.Department of Medical Laboratory Science,Ambrose Alli University,Ekpoma Edo State,Nigeria
}

\begin{abstract}
Differential white blood count in children between the age of 5-10 years in Owerri was investigated in 50 malaria positive children and 20 age matched children who were negative for malaria. Their differential count where estimated in addition with their PCV and platelet. The mean total white blood cell count was also estimated the result obtained shows that The mean total white blood cell count was significantly lower in malaria subjects than in non infected subjects $(5.1 \pm 0.98),(7.9 \pm 1.2),(p<0.05)$ the results obtained also shows that neutrophil count of infected subjects $(42.7 \pm 7.90)$ was significantly lower $(P<0.05)$ when compared with that of non infected subjects (49.0土8.60). The mean value of lymphocyte count of malaria subjects was significantly increased $(p<0.05)(52.5 \pm 9.35)$ when compared with non infected subjects (48.9 98.80$)$. The mean value of eosinophil count of malaria subjects was significantly increased (2.2 \pm 0.49$)$ when compared with non infected subjects (1.1 \pm 0.27$)$. The mean value of monocyte was significantly increased in malaria infected

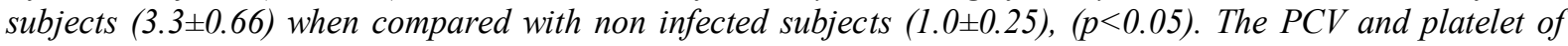
infected subjects respectively were significantly decreased when compared with that of non infected subjects $(p<0.05)$. Conclusively, malaria parasite infection affects almost all the blood cells.
\end{abstract}

Keywords: Malaria, Parasitamia, monocyte, differential count, PCV and platelet.

\section{Introduction \\ MALARIA}

Malaria has been in existence since ancient times and was first described in 4BC, but not until 1889 was the protozoon causing malaria described, and in 1879 female anopheles mosquito was demonstrated to be the vector for the disease. Malaria is an infectious disease caused by the protozoa plasmodium species, it is usually transmitted from one person to another through the bite of an infected female anopheles mosquito, while taking blood meal, thus the female anopheles mosquito acts as the vector . The levels of endemicity of malaria vary from country to country.

Malaria parasitaemia is one of the most important causes of child mortality worldwide, the most frequently encountered in Nigeria and other parts of the world is plasmodium falciparium which annually kills not less than 1Million children in Africa alone, (Snow et al., 2005). This death toll is only one aspect of the global burden of malaria. P.falciparium is estimated to cause about half a billion episode of disease each year (Snow et al., 2005) and there are hundreds of millions of course due to other parasite species-plasmodium vivax, plasmodium malaria, and plasmodium ovale. In region of high malaria transmission, every member of the community might be chronically infected, and in one Gambian village, it was found that about one quarter of all diseases were admitted to the hospital during the first 10 years of life (Snow et al., 2001).

Malaria is the evolutionary driving force behind sickle cell diseases, thalassemia, glucose-6-phosphate deficiency and other erythrocyte defects that together comprise the most common mendelian disease of human kind. HbS, the allele that gives rise to sickle hemoglobin is regarded as the classic parading of balanced polymorphism in human population heterozygote have 10 fold reduced risk of severe malaria. What is remarkable is the range of erythrocyte variant, apart from $\mathrm{HbS}$ that have resulted from evolutionary selection by malaria. They include other variants of the haemoglobin, beta gene-namely $\mathrm{HbC}$ and $\mathrm{HbE}$.

Malaria infection in human is usually associated with a reduction in haemiglobin level frequently leading to anaemia. Plasmodium falciparium causes the most severe and prefund anaemia with a significant risk of death. This cannot be explained simply by the direct destruction of parasitized red blood cells at the time of release of merozoites(Muller et al.2007,), in review, Clara Menendez, alan fleming and pedroalonse focused on recent advances in our knowledge of the path physiology, epidemiology management and prevention of anaemia from falciparum malaria. Anaemia is the major cause of death in malaria infection due to difficulty in diagnosis especially where parasitaemia and the clinical picture may be confused with other causes of anemia. 
Human blood is composed of liquid cellular component namely erythrocytes, Leucocytes and the thrombocytic each of these cells perform a particular function in the human physiology. In health, those cells are adequately produced to a particular range for the sex and age of the individual. In disease there have been records of alteration in the production of the cells and the rate of function they perform. Differential count determines the relative percentage of each type of white cell count as the white cells are made up o five different cells namely neutrophil, lymphocytes, Eosinophil, Monocytes and Basophils. The white cells are the first line of defense in different disease and the percentage of which they appear in different count would give an insight of the kind of infection one may be suffering from. For instance, there have been records of neutrophilia in bacterial infection and neutropenia in other non bacterial related disease: Lymphocytosis, some viral infections and parasitic infection.

Basophiles has to do with blood clotting, monocyte are seen in some bacterial infections and so on (Shah, 2005). Past studies record that malaria parasites attacks the red cell thereby causing their lyses which may result or reduced haemoglobin level and packed cell volume

\section{JUSTIFICATION FOR STUDY}

Differential count gives an insight of what goes on in the body system. It tells more about the level of each of the white cells in the body in health and disease. It also looks at the morphology of the red cells revealing about the appearance of the red cells in terms of size, shape and hemoglobin content. Much work has been documented on red cells but not much has been documented on the effect of malaria on white cells. This work set out to study the level of different white cells in malaria parasitaemia with the aim of determining if they are increased or decreased or not affected when compared with control subjects by malaria which will add to knowledge and assist in the diagnosis and management of malaria infections.

\section{AIMS AND OBJECTIVES}

The aim of this work is to assess the effect malaria may have on some hematological parameter through the following objectives.

1 To determine the effect of malaria parasite on total and differential white cell count.

2 To assess the effect of malaria parasite density on leukocyte numbers.

3 To determine the platelet and PCV of malaria infected children

4 To compare the values obtained with those of non infected subjects.

\section{STUDY AREA}

\section{Materials And Method}

This project was carried out in Owerri in Imo state between the months of November to December. Owerri is located in the tropic rain forest of South east Nigeria, it lies on the $5^{0} 27=50^{0}, 31^{0} \mathrm{~N}$ and longitude $6-58-7^{0}$ OSE. It has a population of about $(75,215,35$, and 278 male and 39937 female) and its comsmoplitan being home to many non indigenes apart from the ethnic Igbo which is a major ethnic group area. The inhabitant is mainly Christian, few Muslims and pagans scattered within the area. The climate of the area is tropical with its mean daily temperature of $29 \pm 50^{\circ \mathrm{c}}$ for most of the year.

\section{STUDY POPULATION}

The subject used for the study were fifty children aged between 5 to 10 years who were diagnosed positive for malaria parasite and 20 aged matched children who were negative for malaria and who served as control subjects.

\section{ETHICAL CLEARANCE}

An introduction letter obtained from the medical laboratory department and detail proposal of the researched work where presented to laboratories, clinics and hospitals whose patients where used to perform the research work. Informed consent was obtained from the parents and relatives of the controlled subjects that gave consent based on the fact that they would be screened free for malaria parasite.

\section{LABORATORY PROCEDURE (BLOOD COLLECTION}

Finger pricking was used to prepare both thick and thin film, two film where made for each investigation, two thick films for concentration of the parasite and two thin films for parasite identification. A blood from the tip of a finger which had being already carefully cleaned with $70 \%$ of ethanol was allowed to dry.

A sterile lancet (micro lance) was used to prink the finger, and the finger was squeezed gentle to obtain a good drop of blood on a clean grease free slide with the Conner of another slide a square or circular patch of moderate thickness was made on the for thick film (it should be possible to read through it) on the other slide a drop of blood that was smaller than the one for thick film was dropped on the slide. The edge of another glass slide was applied in front of the drop of the blood at an angle of $45^{\circ}$, the blood was allowed to spread along its edge. The spreader slide was pushed forward and was kept at an angle to achieve a proper thin film with unbroken layer of red blood cells with the film not touching the edge of the slide. Both slide was allowed to air dry and was been protected from dust, flies and ants before staining. 
The patient name was written at the other end of the slide.

Staining:The thick film was stained by immersing the slide for 20-30 minutes in a staining jar containing, Giemsa stain that was freshly diluted with 1.9 with buffer water. The slide was washed in buffered water for 3 mines.

The slide was stored upright to dry while thin film was first fixed in pure natural for 30 seconds, by immersing it or by putting a few drops of methanol on the slide.

The slide was immersed in a freshly prepared 5\% Giemsa stain solution for 20-30mins. The slide was flushed with tap water to prevent deposition of precipitate on the film.

The slide was allowed to dry vertically (Shapiro et al., 2007).

Examination:Both films were examined using x 100 objective.

\section{Result Analysis}

Table 4.1: showing comparison of the mean values of differential white blood cell counts in malaria patients and control subjects.

\begin{tabular}{|c|c|c|c|}
\hline PARAMETERS & MALARIA PATIENTS & CONTROL SUBJECT & P. VALUE \\
\hline Total WBC $\left(10^{9} / \mathrm{L}\right)$ & $5.1 \pm 0.98$ & $7.9 \pm 1.20$ & $\mathrm{P}<0.05$ \\
\hline Nuetrophil $(\%)$ & $42.7 \pm 7.90$ & $49.0 \pm 8.60$ & $\mathrm{P}<0.05$ \\
\hline Lymphocyte (\%) & $52.5 \pm 9.35$ & $48.9 \pm 8.80$ & $\mathrm{p}<0.05$ \\
\hline Eosinophil (\%) & $2.2 \pm 0.49$ & $1.1 \pm 0.27$ & $\mathrm{P}<0.05$ \\
\hline Monocytes (\%) & $3.3 \pm 0.66$ & $1.0 \pm 0.25$ & $\mathrm{P}<0.05$ \\
\hline
\end{tabular}

In the table above, total WBC and neutrophil counts were significantly decreased in malaria patients when compared with the control subjects $(\mathrm{P}<0.05)$. The table also showed that there was a significantly increased count in lymphocytes, eosinophils and monocytes of the patients when compared with the control $(\mathrm{P}<$ $0.05)$

Table 4.2: showing comparison of platelet counts and PVC level in malaria patients and normal subjects.

\begin{tabular}{|c|c|c|c|}
\hline PARAMETER & MALARIA PATIENT & NORMAL SUBJECTS & P. VALUE \\
\hline Platelet & $174.8 \pm 31.0$ & $269.7 \pm 49.2$ & $\mathrm{P}<0.05$ \\
\hline PCV (\%) & $27.8 \pm 4.96$ & $34.8 \pm 6$ & $\mathrm{P}<0.05$ \\
\hline
\end{tabular}

The table showed that platelet count and PCV level were significantly reduced in malaria patients when compared with normal subjects $(\mathrm{P}<0.05)$.

Table 4.3: Differential count as it relates to parasite Density

\begin{tabular}{|c|c|c|c|c|}
\hline Parasite density & Neutrophil & Lymphocyte & Eosinophil & monocyte \\
\hline $\mathrm{Pd}_{\mathrm{n}}$ " & 39.6 & 54.6 & 1.6 & 4 \\
\hline $\mathrm{Pd}_{6} 2$ & 48.0 & 47.1 & 2.36 & 2.6 \\
\hline $\mathrm{Pd}_{8} 3$ & 42.1 & 52.5 & 2.28 & 3.12 \\
\hline $\mathrm{P}_{11} 4$ & 41.8 & 47.6 & 2.3 & 2.4 \\
\hline
\end{tabular}

\section{Discussion}

In this study some haematological parameters where assessed in children 5-10 years of age suffering from malaria parasitaemia, with the aim of finding out the effect of malaria parasite on the parameters. Results obtained show statistical significant reduction in PCV, the reason could be due to destruction of parasitized red cells which occur in the spleen and this is determined by the degree of parasitaemia. P-falciparum also decreases the erythropoietin response resulting in less erythropoeisis.

There is a reduction in total WBC, this could be as a result of infection which could have affected the bone marrow or due to poor prognosis and chemotherapy etc, and since erythropoiesis is affected there is a tendency of leucopoiesis equally being affected.

There is a reduction in neutrophil count (neutropenia), this could be as a result of decreased level of production of neutrophil or increased removal from the system by macrophage and also parasitic infections such as malaria. It can also be due to some commonly used anti-malaria drugs. This agrees with the previous report by (Chenl et al., 2005).

There is slight increase in monocyte (monocytosis), which usually goes with infectious protozoal diseases (malaria, kala-azar, tryponosomiasis) and other diseases such as rickettsial (Rocky Mountain spotted fever, typhus). Bacterial infection (Syphillis). There is an increase in lymphocyte (Lymphocytosis), this could be as a result of infection which could occur after initiation of anti-plasmodial treatment and in acute malaria. This is in line with previous report by $\mathrm{K}$. Lauenbery et al. there is a decrease in platelet which is called thrombocytopenia which could be as a result of peripheral destruction, excessive sequestration use of platelet which is associated with the disseminated intravascular coagulation phenomenon which can occur in malaria infection in. 
Basophil was not seen which could be due to the fact that they result are low in blood usually not medically significant. There is a slightly increase in eosinophil, this could be due to resulting from allergic, parasitic infection.

\section{Conclusion}

At the end of this research was observed that malaria parasite affected all the parameter Estimated: PCV, Total WBC, Eosinophil, monocyte, Lymphocyte, Neutrophil. Some of the parameters were increased like Lymphocytes, Eosinophil and Monocte while some were decreased e.g. total WBC count, Neutrophil, Platelet and PCV. Conclusively malaria is a serious infection which could affect haemopoiesis and other systemic problems.

\section{References}

[1]. Snow, R.W., Guera, C.A., Noov, A.M., Myint, H.Y.,and Hays, (2005): The Global Distribution Of Clinical Episodes Of Plasmodium Falciparium Malaria. Nature 434: 214-217.

[2]. Snow, R.N., Trapeyf, and Marsh, K., (2001): The Past Present And Future Of Childhood Malaria Mortality In Africa Treads .Parasitol. 17 (12): 593

[3]. Chenl, Zhang,and Sendo, F. (2005): Function Of Neutrophil In Experimental Cerebral Malaria. Clin. Exp. Immunal. 120: 12-33

[4]. Muller, Immerman, PA., Reader, J.C., (2007). Plasmodium Malaria And Plasmodium Oral. The Bashful Malaria Parasite.Tread in parasitology 23(6): 278-83. 